\title{
Hypoalbuminemia, systemic inflammatory response syndrome, and functional outcome in intracerebral hemorrhage
}

DOI:

10.1016/j.jcrc.2017.06.002

\section{Document Version}

Accepted author manuscript

Link to publication record in Manchester Research Explorer

Citation for published version (APA):

MNEMONICH Investigators (2017). Hypoalbuminemia, systemic inflammatory response syndrome, and functional outcome in intracerebral hemorrhage. Journal of Critical Care, 41, 247-253.

https://doi.org/10.1016/j.jcrc.2017.06.002

Published in:

Journal of Critical Care

\section{Citing this paper}

Please note that where the full-text provided on Manchester Research Explorer is the Author Accepted Manuscript or Proof version this may differ from the final Published version. If citing, it is advised that you check and use the publisher's definitive version.

\section{General rights}

Copyright and moral rights for the publications made accessible in the Research Explorer are retained by the authors and/or other copyright owners and it is a condition of accessing publications that users recognise and abide by the legal requirements associated with these rights.

\section{Takedown policy}

If you believe that this document breaches copyright please refer to the University of Manchester's Takedown Procedures [http://man.ac.uk/04Y6Bo] or contact uml.scholarlycommunications@manchester.ac.uk providing relevant details, so we can investigate your claim.

\section{OPEN ACCESS}




\section{Accepted Manuscript}

Hypoalbuminemia, systemic inflammatory response syndrome, and functional outcome in intracerebral hemorrhage

Mario Di Napoli, Réza Behrouz, Christopher H. Topel, Vivek Misra, Fulvio Pomero, Alessia Giraudo, Paolo Pennati, Luca Masotti, Floris H.B.M. Schreuder, Julie Staals, Catharina J.M. Klijn, Craig J. Smith, Adrian R. Parry-Jones, Mark A. Slevin, Brian Silver, Joshua Z. Willey, Mahmoud R. Azarpazhooh, Jaime

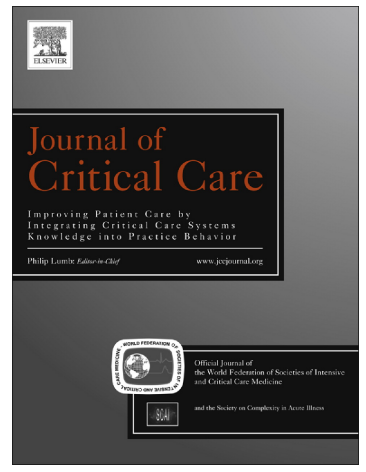
Masjuán Vaelljo, Hipólito Nzwalo, Aurel Popa-Wagner, Daniel A. Godoy, the MNEMONICH Investigators

PII: S0883-9441(17)30103-X

DOI: doi: $10.1016 /$ j.jcrc.2017.06.002

Reference: YJCRC 52550

To appear in:

Revised date:

Accepted date: \#\#\#ACCEPTEDDATE\#\#\#

Please cite this article as: Mario Di Napoli, Réza Behrouz, Christopher H. Topel, Vivek Misra, Fulvio Pomero, Alessia Giraudo, Paolo Pennati, Luca Masotti, Floris H.B.M. Schreuder, Julie Staals, Catharina J.M. Klijn, Craig J. Smith, Adrian R. Parry-Jones, Mark A. Slevin, Brian Silver, Joshua Z. Willey, Mahmoud R. Azarpazhooh, Jaime Masjuán Vaelljo, Hipólito Nzwalo, Aurel Popa-Wagner, Daniel A. Godoy, the MNEMONICH Investigators, Hypoalbuminemia, systemic inflammatory response syndrome, and functional outcome in intracerebral hemorrhage, (2017), doi: 10.1016/j.jcrc.2017.06.002

This is a PDF file of an unedited manuscript that has been accepted for publication. As a service to our customers we are providing this early version of the manuscript. The manuscript will undergo copyediting, typesetting, and review of the resulting proof before it is published in its final form. Please note that during the production process errors may be discovered which could affect the content, and all legal disclaimers that apply to the journal pertain. 


\section{Hypoalbuminemia, Systemic Inflammatory Response Syndrome, and Functional Outcome in Intracerebral Hemorrhage}

Mario Di Napoli, MD ${ }^{1}$; Réza Behrouz, DO, $\mathrm{PhD}^{2}$; Christopher H. Topel, $\mathrm{DO}^{2}$; Vivek Misra, MBBS, $\mathrm{MD}^{3}$; Fulvio Pomero, $\mathrm{MD}^{4}$; Alessia Giraudo, $\mathrm{MD}^{4}$; Paolo Pennati, $\mathrm{MD}^{4,5}$; Luca Masotti, $\mathrm{MD}^{6}$; Floris H.B.M. Schreuder, $\mathrm{MD}^{7,8}$; Julie Staals, MD, $\mathrm{PhD}^{7}$; Catharina J.M. Klijn, MD, $\mathrm{PhD}^{8}$; Craig J Smith, MBChB, MD, MRCP ${ }^{9}$; Adrian R Parry-Jones, MBChB, MRCP, $\mathrm{PhD}^{8}$; Mark A. Slevin, PhD, FRCPath ${ }^{10}$; Brian Silver, MD ${ }^{11}$; Joshua Z. Willey, MD, MS ${ }^{12}$; Mahmoud R. Azarpazhooh, MD ${ }^{13,14}$; Jaime Masjuán Vaelljo, MD, PhD ${ }^{15}$; Hipólito Nzwalo, MD ${ }^{16}$; Aurel Popa-Wagner, MD, PhD ${ }^{17,18}$; Daniel A. Godoy, $\mathrm{MD}^{19}$; the MNEMONICH Investigators

1. Neurological Service, San Camillo de' Lellis General Hospital, Rieti; and the Neurological Section, Neuro-epidemiology Unit, SMDN, Centre for Cardiovascular Medicine and Cerebrovascular Disease Prevention, Sulmona, L'Aquila, Italy

2. Department of Neurology, School of Medicine, University of Texas Health Science Center, San Antonio, Texas, USA

3. Houston Methodist Hospital, Houston, Texas, USA

4. Department of Internal Medicine, Santa Croce and Carle Hospital, Cuneo, Italy

5. Emergency-Urgency Department, Livorno Hospital, Livorno, Italy

6. Department of Internal Medicine, Santa Maria Nuova Hospital, Florence, Italy

7. Department of Neurology, Maastricht University Medical Center, Maastricht, the Netherlands

8. Department of Neurology, Donders Institute for Brain, Cognition and Behaviour, Centre for Neuroscience, Radboud University Nijmegen Medical Centre, Nijmegen, the Netherlands

9. Stroke and Vascular Centre, Institute of Cardiovascular Sciences, University of Manchester, Manchester Academic Health Science Centre, Salford Royal NHS Foundation Trust, Stott Lane, Salford, $U K$

10. School of Healthcare Science, Manchester Metropolitan University, Manchester, UK

11. Department of Neurology, University of Massachusetts Medical School, Worcester, Massachusetts, USA

12. Department of Neurology, Columbia University College of Physicians \& Surgeons, New York, NY, USA 
13. Department of Neurology, Ghaem Hospital, Mashhad University of Medical Sciences, Mashhad, Khorasan Razavi, Iran

14. Department of Clinical Neurological Sciences, Western University of London, Ontario, Canada

15. Hospital Universitario Ramón y Cajal, Universidad de Alcalá, Madrid, Spain

16. Stroke Unit, Centro Hospitalar do Algarve, University do Algarve, Algarve, Portugal

17. University of Medicine and Pharmacy Craiova, Romania

18. Department of Psychiatry, Universitätsmedizin Rostock, Rostock, Germany

19. Neurocritical Care Unit, Sanatorio Pasteur, and Unidad de Terapia Intensiva Hospital Interzonal de Agudos "San Juan Bautista" Av Moreno e Illia, Catamarca, Argentina

\section{CORRESPONDENCE}

Mario Di Napoli, MD - Neurological Section, SMDN-Center for Cardiovascular Medicine and Cerebrovascular Disease Prevention, Via Trento, 41, 67039-Sulmona, L'Aquila, Italy.

E-mail mariodinapoli@katamail.com

\section{KEY WORDS}

Intracerebral hemorrhage; Albumin; Hypoalbuminemia; Inflammation; Systemic inflammatory response syndrome; Stroke outcomes

\section{WORD COUNT}

Abstract $=242$

Text Word count $=3170$

Figures: 2

Tables: 3

References $=40$

Supplemental material: Table e1; Figure e1

\section{FINANCIAL SUPPORT}

This project was supported by each participating center's institutional/departmental funds.

C.J.M. Klijn is supported by a clinical established investigator grant from the Dutch Heart Foundation (2012T077), and an ASPASIA grant from The Netherlands Organisation for Health Research and Development, ZonMw (grant number 015008048). Adrian Parry-Jones is funded by an NIHR Clinician Scientist Award.

\section{DISCLOSURES}

All authors have disclosed that they do not have any conflicts of interest. 


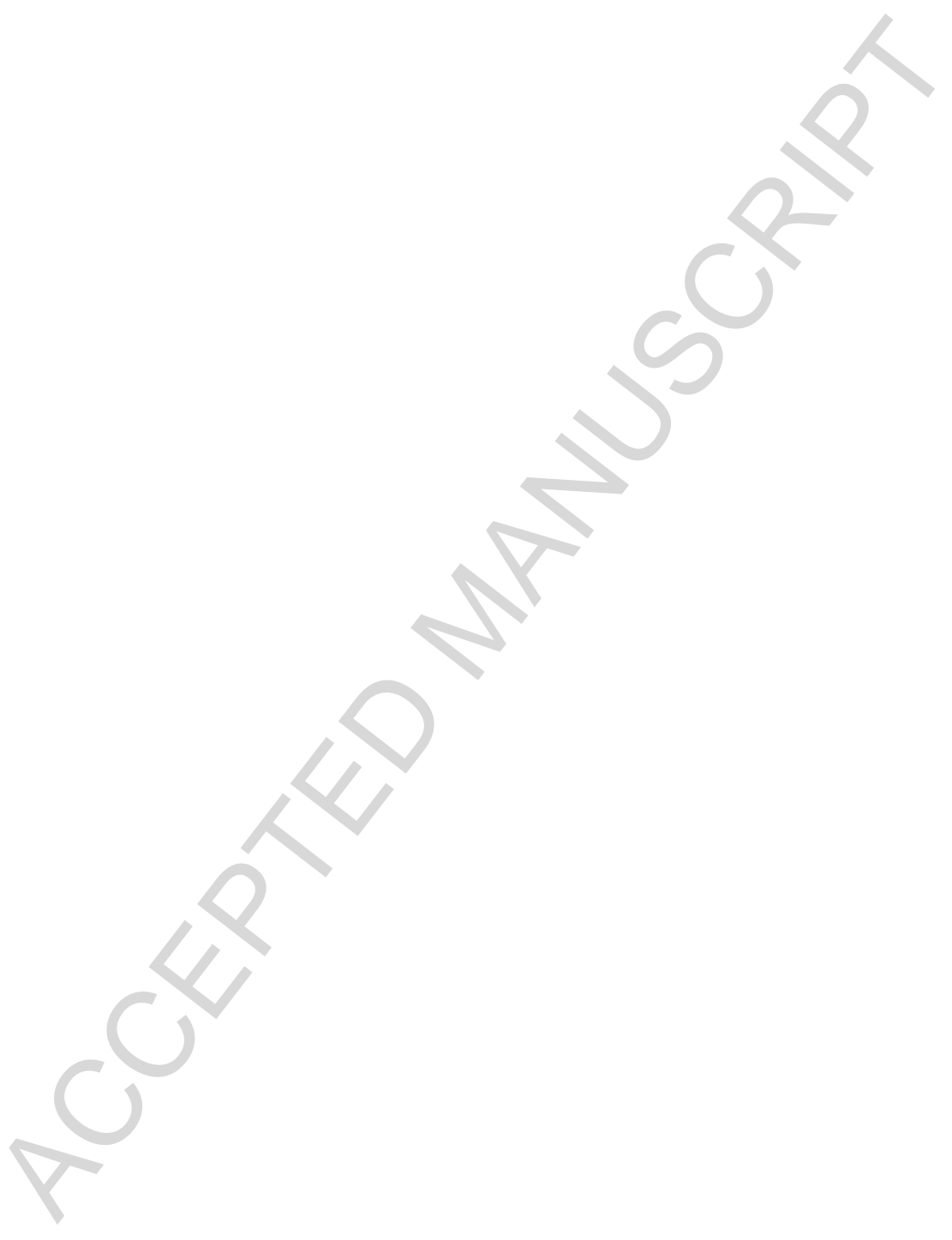




\section{ABSTRACT}

Purpose: Hypoalbuminemia and systemic inflammatory response syndrome (SIRS) are reported in critically-ill patients, but their relationship is unclear. We sought to determine the association of admission serum albumin and SIRS with outcomes in patients with intracerebral hemorrhage (ICH).

Methods: We used a multicenter, multinational registry of ICH patients to select patients in whom SIRS parameters and serum albumin levels had been determined on admission. Hypoalbuminemia was defined as the lowest standardized quartile of albumin; SIRS according to standard criteria. Primary outcomes were modified Rankin Scale (mRS) at discharge and in-hospital mortality. Regression models were used to assess for the association of hypoalbuminemia and SIRS with discharge mRS and in-hospital mortality.

Results: Of 761 ICH patients included in the registry 518 met inclusion criteria; 129 (25\%) met SIRS criteria on admission. Hypoalbuminemia was more frequent in patients with SIRS (42\% versus 19\%; p $<0.001$ ). SIRS was associated with worse outcomes (OR: 4.68, 95\%CI, 2.52-8.76) and in-hospital allcause mortality (OR: $2.18,95 \% \mathrm{CI}, 1.60-2.97)$, while hypoalbuminemia was not associated with allcause mortality.

Conclusions: In patients with ICH, hypoalbuminemia is strongly associated with SIRS. SIRS, but not hypoalbuminemia, predicts poor outcome at discharge. Recognizing and managing SIRS early may prevent death or disability in ICH patients. 


\section{INTRODUCTION}

Hypoalbuminemia is common in critical illness but its role in outcomes has not been as well established.[1] It may have multiple causes, including malnutrition, acute and chronic inflammation, infection, renal disease, and is associated with poor overall health and frailty. It is also a recognized prognostic factor in multiple acute life threatening injuries.[2-4] In acute ischemic stroke, hypoalbuminemia is associated with poor outcome,[5-8] and is a strong predictor of early death in subarachnoid hemorrhage. $[9,10]$ Little is known about the significance of serum albumin levels as a prognostic factor in spontaneous intracerebral hemorrhage (ICH).[11]

ICH induces an acute-phase inflammatory response (APR).[12] APR is a cytokine-driven process that can determine a host's systemic inflammatory response syndrome (SIRS). Approximately $20 \%$ of patients with ICH develop SIRS with an increased risk of poor functional outcome at discharge.[13] Patients with APR often have a decrease in serum albumin concentration, which is primarily caused by reduced albumin liver synthesis and albumin passage from the vascular into the extravascular space due to enhanced endothelial permeability. $[3,14]$ Whether low albumin is a marker of a severe systemic inflammatory response or independently associated with outcome in ICH patients is unknown.

The aim of this study was to compare the frequency of hypoalbuminemia in ICH patients with or without SIRS and to test the hypothesis that initial serum albumin is independently associated with poor functional outcome in patients with ICH. The secondary objectives were to examine the relationship between SIRS and albumin levels in ICH patients, and test interaction between SIRS and serum albumin levels in predicting functional outcome at discharge and in-hospital mortality. 


\section{MATERIALS \& METHODS}

\section{Patient Population}

The study population consisted of patients registered in the Multi-National survey on Epidemiology, Morbidity and Outcomes in Intracerebral Hemorrhage (MNEMONICH) with data extracted from the registry on December 31, 2015. MNEMONICH (NCT2567162 ClinicalTrials.gov) is an ongoing, international, multicenter, observational, collaborative database of consecutive patients aged $\geq 18$ years with spontaneous ICH from participating centers in Europe, South America, and the United States.[15,16] Details on participating centers, study period, demographic and laboratory data are given in Supplemental Table 1. In MNEMONICH, spontaneous ICH is defined as acute brain intraparenchymal bleeding confirmed by computed tomography (CT) scan, in the absence of an underlying cause other than small vessel disease. Patients with anticoagulantassociated ICH are also included. Bleeding due to brain tumors, vascular malformations, aneurysms, and trauma are excluded. Registered data included demographics, clinical, laboratory, and neuro-radiological findings collected at the participating centers. Data quality and consistency are regularly monitored by the coordinating center (Rieti, Italy). All ICH patients included in MNEMONICH are managed according to local guidelines, based on either the guidelines of the American Heart Association/American Stroke Association or the European Stroke Organization.[17-19] Approval by the local Ethics Committee was obtained by each center prior to participation in MNEMONICH.

\section{Inclusion criteria}

Consecutive patients with ICH enrolled in MNEMONICH were considered eligible for the current analysis based on the following criteria: (1) spontaneous ICH presenting within 24 hours of symptom onset confirmed on CT of sufficient quality for calculation of ICH volume; (2) a blood sample with 
albumin determination within 24 hours after ICH onset; and (3) complete follow-up on December 31, 2015. We excluded patients who had undergone hematoma evacuation during their acute admission due to the potential confounding effect of surgery.

\section{Clinical and Radiological Variables}

Clinical data including age, sex, past medical history, and previous medication use (including oral anticoagulants and antiplatelet medications) were all collected through interviews with patients or their relatives. Prospectively recorded admission variables included Glasgow Coma Scale (GCS), systolic and diastolic blood pressure, blood glucose, ICH score, and time from symptom onset to CT. All definitions and coding are based on the NINDS common data elements (https://www.commondataelements.ninds.nih.gov/Stroke.aspx\#tab=Data_Standards).

At each center, experienced neurologists or neuro-radiologists blinded to clinical data, functional outcome, and albumin results assigned ICH locations for all patients as lobar, deep, brain stem, or cerebellar. Volumetric hematoma measurements were performed according to ABC/2 method by local investigators.[20] To determine inter-rater reliability of the measurements for all investigators, a random sample of $19 \mathrm{CT}$ scans were also analyzed by a central reader (MDN, Rieti, Italy). Finally, intraventricular extension of hemorrhage (IVH) was determined as categorical variable (present or absent).

\section{Serum Albumin Determination}

At each center, serum albumin levels were analyzed by the local clinical laboratory using different analytical methods (bromocresol purple dye-binding in 3, bromocresol green dye-binding method in 1 center). Because assay methods have a marked effect on the concentration results mainly in 
hypoalbuminemic patients,[21] albumin values were standardized calculating the Z-score value for each independent cohort, by subtracting the mean and dividing by the standard deviation, and adding 3 units to each value in order to avoid negative results.

\section{Systemic Inflammatory Response Syndrome (SIRS)}

In the present analysis, we considered only occurrence of SIRS at admission. SIRS was defined according to standard criteria as 2 or more of the following: (1) temperature of more than $38^{\circ} \mathrm{C}$ $\left(100.4^{\circ} \mathrm{F}\right)$ or less than $36^{\circ} \mathrm{C}\left(96.8^{\circ} \mathrm{F}\right)$, (2) heart rate $(\mathrm{HR})$ of more than 90 beats per minute, (3) respiratory rate $(\mathrm{RR})$ of more than 20 breaths per minute or arterial carbon dioxide tension $\left(\mathrm{PaCO}_{2}\right)$ of less than $32 \mathrm{~mm} \mathrm{Hg}$, and (4) WBC count of $>12,000 / \mu \mathrm{L}$ or $<4,000 / \mu \mathrm{L}$ or $>10 \%$ band forms.[22] All patients were strictly monitored during the first 7 days after admission to identify any clinical sign suggestive of potentially clinical infection to explain SIRS at admission. In the presence of clinical suspicion of infection, culture results were obtained from different sources. The physicians directly in charge of the patient evaluated each positive culture result to determine whether it represented true infection or merely contamination and as a possible cause of SIRS at admission. This decision was based on multiple factors, including the patient's history, physical examination, radiological and microbiological and laboratory findings. The admission SIRS group was divided into those with culture results obtained from any site that were positive for bacterial growth (culture-positive) and those without any evidence of infectious etiology (culture-negative).

\section{Definition of Endpoints}

The primary outcome was the modified Rankin Scale (mRS) score at final discharge.[23] $\mathrm{mRS}$ is a stroke outcome scale with scores ranging from 0 (no symptoms at all) to 6 (dead). A score of 2 or less 
indicates functional independence. The secondary endpoint for the study was in-hospital all-cause mortality, with censoring at the time of hospital discharge.

\section{Statistical Analysis}

Data are presented as mean \pm standard deviation (SD) for normally distributed continuous variables, median and interquartile range (IQR) for non-normally distributed continuous variables, and as frequencies for categorical variables. Albumin levels were also categorized in quartiles based on standardized albumin levels. Low albumin or hypoalbuminemia was defined as levels below the lowest quartile. Baseline characteristics between patients with and without hypoalbuminemia were compared using independent samples t test, $\chi^{2}$, and Mann-Whiney, or Kruskal-Wallis test as appropriate.

Inter-rater reliability between centers in using $\mathrm{ABC} / 2$ method was measured with intra-class correlation coefficients (ICC) with the use of analysis of variance.[24] An intra-class correlation coefficient of 1 demonstrates perfect reliability, and $>0.8$ represents excellent reliability. Multifactorial general linear regression models were used to analyze the association between hypoalbuminemia with SIRS and baseline hematoma volume, with standardized albumin concentrations as a dependent variable and included sex, infratentorial hematoma, intraventricular hemorrhage, and SIRS on admission as binary variables. Baseline ICH volume, International Normalization Ratio (INR), and glucose at admission were log-transformed to approximate the normal distribution, and together with age and GCS score were included as continuous variables in the model. 
We assessed the association of hypoalbuminemia with outcome using $\mathrm{mRS}$ with a multivariable ordinal logistic regression model because $\mathrm{mRS}$ is an ordinal categorical variable.[25] We estimated the adjusted common odds ratio (OR) for a shift in the direction of better outcome on the mRS, and reported the results as unadjusted and adjusted OR with 95\% confidence intervals (CI). Adjustment was made for pre-specified baseline characteristics known to be associated with worse outcome in ICH, including age, log-transformed ICH volume, log-transformed INR, log-transformed glucose at admission, and GCS as continuous variables, and sex, infratentorial location, intraventricular extension, and SIRS at admission as binary variables. Finally, a multivariable logistic regression was performed to estimate adjusted OR for in-hospital mortality and included formal testing for interaction between albumin and SIRS at admission. Multivariable analysis included variables found to be significantly different $(\mathrm{p}<0.05)$ between albumin groups and with potential to influence mortality outcome as used in primary analysis.

\section{RESULTS}

Of the 16 centers participating in MNEMONICH registry on December 31, 2015, 5 centers were able to send patient data to Coordinating Center by June 30, 2016 on time for locking central database: 761 ICH patients were included. In 1 center, albumin concentration was not routinely assessed and all patients $(n=175)$ were excluded. Of the remaining 586 patients, $518(88.4 \%)$ had serum albumin levels measured on admission. Patients without baseline serum albumin levels $(n=68,11.6 \%)$ were similar to patients with baseline serum albumin determinations except they were significantly older ( $72 \pm 13$ vs $68 \pm 13 ; \mathrm{p}=0.047)$ and less often had arterial hypertension (58\% vs $81 \%$; $\mathrm{p}<0.001)$. 


\section{Albumin and Baseline Patient Characteristics}

The study cohort had a mean age of $68 \pm 13$ years (range: 25 to 95 years), and $60.4 \%(n=313)$ were men. The median GCS was 13 (IQR: 7-14) and median ICH score was 2 (IQR: 1-3). Median hematoma volume on the first CT scan was $18 \mathrm{~mL}$ (IQR: 9-36.5 mL). There was excellent interrater reliability between local and central measurement of $\mathrm{ICH}$ volume using the $\mathrm{ABC} / 2$ technique (ICC: 0.91, 95\%CI: 0.84-0.96). Bromocresol green dye-binding method was mainly used for assaying albumin concentration (69\%). There were significant differences in albumin levels between cohorts (Supplemental Figure S1) and albumin values were standardized for data analysis. Median standardized albumin concentration was 3.14 (IQR: 2.3-3.8). Baseline characteristics of the study cohort are presented in Table 1.

\section{Albumin and SIRS Occurrence on Admission}

SIRS was present in $129(25 \%)$ patients on admission, was significantly associated with hypoalbuminemia (Table 1), and differed between standardized albumin quartiles. SIRS was present in $42 \%$ of patients in the lowest albumin quartile while only in $11 \%$ of patients in the highest quartile $\left(\mathrm{p}<0.001, \chi^{2}\right.$ for trend; Figure 1a). There was an inverse significant relationship between mean standardized albumin concentration and the number of positive SIRS criteria $(\mathrm{p}<0.001$, KruskalWallis test; Figure 1b). In the multifactorial generalized linear regression model, SIRS remained significantly associated with albumin concentration together with female sex, GCS and infratentorial site of hemorrhage (Table 2). Of 129 patients with SIRS at admission, 99 (78\%) had negative culture results during 7 days follow-up, $8(6 \%)$ had positive cultures, and culture data were not available in $22(17 \%)$ patients. Standardized albumin concentrations were not different $(p=0.6)$ in patients with SIRS at admission and positive culture versus negative culture patients. 


\section{Albumin, SIRS, and Hematoma Volume}

Standardized albumin concentration was significantly higher in patients with hematoma volumes lower than $30 \mathrm{~mL}$ [3.4 (IQR: 2.6-3.9)] when compared to patients with hematoma volume between $30-60 \mathrm{~mL}$ [2.8 (IQR: 2.1-3.6), p=0.003] and to patients with hematoma volume >60 mL [2.6 (IQR: 1.9-3.4), $\mathrm{p}<0.001]$. SIRS was significantly $\left(\mathrm{p}<0.001, \chi^{2}\right.$ for trend) more frequent in patients with a greater hematoma ranging from $11.1 \%(n=39 / 351)$, in patients with hematoma volumes lower than $30 \mathrm{~mL}$, to $44 \%(\mathrm{n}=37 / 84)$ in those with hematoma volume between $30-60 \mathrm{~mL}$, to $63.4 \%(\mathrm{n}=52 / 82)$ in those with hematoma volume $>60 \mathrm{~mL}$. In the multifactorial generalized linear regression model, SIRS remained significantly associated with greater hematoma volume (OR: 1.45 95\%CI: 1.33-1.55) together with GCS (OR: 0.90 95\%CI: 0.88-0.93), higher GCS score punctuation (OR: 0.90, 95\%CI: 0.88-0.93), infratentorial site of hemorrhage (OR: 2.26 95\%CI: 1.81-2.81) and higher INR values (OR: 1.36, 95\%CI: 1.09-1.69), but not with standardized concentration of albumin (OR:0.99, 95\%CI: 0.92-1.08).

\section{Albumin, SIRS and Functional Outcome at Discharge}

The median length-of-stay was 8 days (IQR: 5-12). In univariable ordinal regression analysis, standardized hypoalbuminemia was significantly associated with worse outcomes in the overall distribution of mRS scores (common OR: 2.52, 95\%CI, 1.73-3.68). After adjustment for age, sex, ICH volume, GCS, infratentorial location, INR at admission, serum glucose, SIRS occurrence at admission, and IVH, standardized hypoalbuminemia was no longer significantly associated with the overall distribution of $\mathrm{mRS}$ score at discharge (adjusted common OR: 0.82, 95\%CI, 0.52-1.29). The presence of SIRS on admission was significantly associated with worse discharge outcome in the multivariable analysis (adjusted common OR: 4.68, 95\%CI, 2.518.76; Figure 2). SIRS patients on admission had significantly worse median mRS scores on discharge (6 versus 3, $\mathrm{p}<0.001)$. 


\section{Albumin, SIRS and Mortality}

There were $172(33 \%)$ in-hospital deaths. In-hospital death was significantly more frequent in patients with low albumin, compared to those with normal albumin $(52 \%$ vs $27 \%, \mathrm{p}<0.001 \log$-rank test; HR 2.24, 95\%CI, 1.65-3.05). For each unit increase of standardized albumin concentration (equivalent to one standard deviation), there was a reduction of around one-third in the death rate (HR: 0.61, 95\%CI, 0.52-0.71). SIRS on admission (adjusted OR: 5.52, 95\%CI, 2.29-13.32) but not standardized hypoalbuminemia (adjusted OR: 1.30, 95\%CI, 0.50-3.36) independently predicted all-cause in-hospital mortality, after adjustment for multiple risk factors. There was no interaction $(\mathrm{p}=0.7)$ between albumin levels and SIRS occurrence at admission (Table 3).

\section{DISCUSSION}

Our study demonstrates that: (1) ICH patients have frequently low concentration of albumin on admission; (2) almost one fourth of the patients with ICH in our multicenter, cohort study had evidence of SIRS at admission; (3) hypoalbuminemia is associated with the presence and severity of SIRS but not independently with functional outcome at discharge and in-hospital mortality, and (4) albumin represents a biomarker of the acute inflammatory response and its severity after ICH.

Albumin is a hepatic protein, and its plasma concentration is controlled by several factors, including rate of albumin synthesis, catabolic rate, albumin distribution, and exogenous albumin loss. Synthesis of albumin is affected by nutritional intake, colloid oncotic pressure variations, and the presence of systemic inflammation [26,27]. When using albumin concentrations as an outcome measure, serum albumin should be compared using a standardized measure. However, a number of different assay methods are used for measuring albumin and these methods give different results.[28] For making our results comparable and generalizable our results, we used standardized concentrations. 
This allows us to generalize our results in different populations and using different assay methods. In clinical practice, the use of local normal range should be encouraged to take account of different assay methods.

In our cohort median albumin concentration was notably lower (Supplemental Figure 1) when compared with the reference range for normal serum albumin (35-55 g/L).[29] By these reference values, several patients that would normally be classified with hypoalbuminemia clinically in other medical conditions may be not correctly classified in the presence of ICH. The distribution curve of ICH patients has an IQR of albumin that is generally less than in normal subjects. This observation has important implications and should be taken in account because these lower values could perhaps over evaluate the negative prognostic meaning of hypoalbuminemia in ICH patients. Standardization of albumin values would resolve this problem.

In our ICH cohort, hypoalbuminemia was associated with SIRS. Decreased serum albumin levels were registered in more than $40 \%$ of SIRS-positive patients and are probably due to diminished liver synthesis,[14] besides functional changes in vascular permeability,[30] with enhanced albumin endothelial passage into the interstitial space.[14,30] In our opinion, the hypoalbuminemia during SIRS probably occurs in a two-stage process. The release of cytokines induced by SIRS (e.g. interleukin-1 and tumor necrosis factor- $\alpha$ ) may cause an acute reduction of albumin during the first 24-48 hours after the acute event that is independent from preexistent nutritional status and nutritional supplementation.[30,31] Initially, interleukin-1 causes an increase in endothelial permeability, with transendothelial loss of albumin. This would explain the hypoalbuminemia observed on admission in these patients. The severity of ICH and increased hematoma volume has been correlated with increased permeability of the BBB.[32] Notably, in our cohort the percentage of 
patients with hypoalbuminemia was 2.5 fold higher in the large volume (>60 mL) group than those in the low volume (<30 mL) group (Table 1). Subsequently, a decrease in production of albumin occurs because of the known depressive effect of interleukin-1 for the mRNA for albumin.[14] Patients with hypoalbuminemia in our cohort were also more likely to have lower GCS scores, infratentorial hematomas, or additional conditions potentially associated with more severe inflammatory response after ICH.[33]

Furthermore, this study showed that admission SIRS in ICH patients is an independent predictor of poor outcome, including disability and mortality, supporting the theory that inflammatory mechanisms play a detrimental role in progression of ICH-induced brain injury.[34] The inflammatory response that follows ICH involves enzyme activation, mediator release, inflammatory cell migration, glial activation, and brain tissue breakdown.[34] When the inflammatory response becomes uncontrolled, SIRS signals the inability of anti-inflammatory mechanisms to down-regulate a profound state of inflammation. This unrestrained inflammation contributes to secondary brain injury, long after the initial insult. Our results highlight the deleterious impact of SIRS on outcome after ICH. Despite controlling for other known prognostic factors, SIRS remained a strong contributor to poor outcomes at discharge and in-hospital mortality. SIRS, therefore, does not seem to be only a marker of severe hemorrhages, but rather contributes independently to outcomes.

To the best of our knowledge, our study is the first to investigate the relationship between SIRS and serum albumin levels in ICH patients. The strong association between hypoalbuminemia and SIRS suggests they may have common pathophysiologic mechanisms. Hypoalbuminemia is likely to be a component of a complex acute systemic inflammatory reaction and interacting physiological systems that together influence outcomes and survival in patients with ICH. In our study, hypoalbuminemia 
was not independently associated with mortality or functional outcome when including SIRS in our regression models, suggesting that levels of albumin may simply be a marker of inflammation and not a possible independent therapeutic target in $\mathrm{ICH}$.

In recent years, there have been attempts to study the utility of albumin use in patients with $\mathrm{ICH}$, based on evidence of multiple neuroprotective effects demonstrated in animal models of ICH and ischemic stroke. In rat models of experimental $\mathrm{ICH}$, treatment with albumin improved neurological function and maintained integrity of the blood-brain barrier without affecting hematoma volume or edema.[35-37] However, despite the positive results of albumin in ICH animal models, clinical trials in acute ischemic stroke (ALIAS),[38] and in ICH [Albumin for ICH Intervention (ACHIEVE), NCT00990509] have shown negative or inconclusive results with concerns for an excess of side effects (pulmonary edema and a small excess of hemorrhagic stroke in the albumin group). Additionally, a meta-analysis of 55 randomized controlled trials as well as a Cochrane systematic review concluded that there was no evidence that albumin administration reduces mortality in critically ill patients.[39,40] These data support our observation that hypoalbuminemia represents a biomarker of SIRS rather than an independent pathologic determinant.

Our study had several limitations. We analyzed data from an existing database and therefore, it is possible that certain preexistent chronic inflammatory conditions or comorbidities and complications (cardiopulmonary, liver disease, etc.) that could have potentially influenced the association with the presence of SIRS criteria or that could have potentially influenced outcomes were not incorporated into the analyses. However, the prevalence of these complications was probably not high enough to substantially influence our results. Furthermore, the association with intrinsic factors of severity such as the GCS and ICH location reinforces a hyper-acute pathophysiological process causing 
hypoalbuminemia. We only included patients who had serum albumin measured at baseline and only medically treated patients, which reduced our cohort size and could have excluded patients with characteristics that contributed to mortality, but were not integrated in our analyses. Moreover, this could have introduced the potential for selection bias in the final study cohort. Residual measured and unmeasured confounding variables may also explain the observed relationship. Serum albumin sampling was assessed on admission and subsequently, we could not determine if timing of albumin concentration and changes over time played a more powerful role in predicating outcomes. Because albumin is an acute phase reactant, it is possible hypoalbuminemia later in the course of hospitalization is a better predictor of outcome than concentrations within the first 24 hours of admission. Further prospective studies with serial measurement of albumin in ICH patients will be required to better understand this. Other serum markers of inflammation, such as cytokines or chemokines, would have helped to further delineate the pathophysiological relationship between albumin inflammation and mortality, but were not available. Finally, we did not analyze ICH etiology and its relationship with hypoalbuminemia.

\section{CONCLUSION}

Hypoalbuminemia is a marker of SIRS in ICH. ICH patients with hypoalbuminemia have a 2-fold increased risk of worse outcome and in-hospital death, compared to those without. However, this predictive effect was not evident when the presence of SIRS was taken in account. Serum albumin measurement may serve as a marker for identifying SIRS-positive ICH patients who are at increased risk of poor functional outcome and in-hospital death, but may not be a therapeutic target. Although further investigation into the underlying mechanism of hypoalbuminemia is warranted, administration of albumin appears unlikely to be beneficial and more data are needed before further evaluation in a randomized trial. 


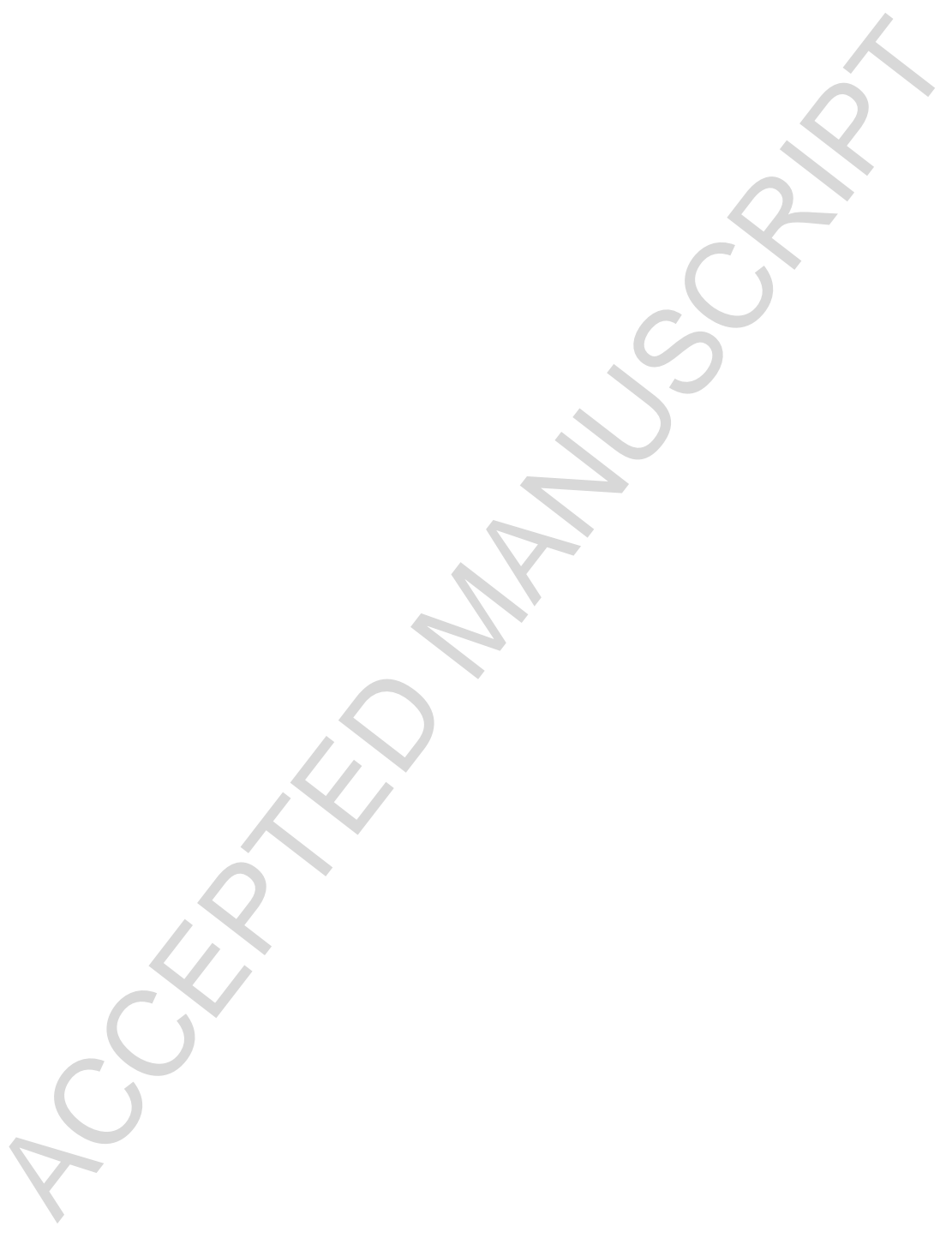




\section{REFERENCES}

1. Caironi P, Langer T, Gattinoni L. Albumin in critically ill patients: the ideal colloid? Current opinion in critical care 2015;21(4):302-8.

2. Moshage HJ, Janssen JA, Franssen JH, Hafkenscheid JC, Yap SH. Study of the molecular mechanism of decreased liver synthesis of albumin in inflammation. The Journal of clinical investigation 1987;79(6):1635-41.

3. Vincent JL, Dubois MJ, Navickis RJ, Wilkes MM. Hypoalbuminemia in acute illness: is there a rationale for intervention? A meta-analysis of cohort studies and controlled trials. Annals of surgery 2003;237(3):319-34.

4. Nicholson JP, Wolmarans MR, Park GR. The role of albumin in critical illness. Br J Anaesth 2000;85(4):599-610.

5. Cho YM, Choi IS, Bian RX, Kim JH, Han JY, Lee SG. Serum albumin at admission for prediction of functional outcome in ischaemic stroke patients. Neurological sciences : official journal of the Italian Neurological Society and of the Italian Society of Clinical Neurophysiology 2008;29(6):445-9.

6. Dziedzic T, Pera J, Slowik A, Gryz-Kurek EA, Szczudlik A. Hypoalbuminemia in acute ischemic stroke patients: frequency and correlates. European journal of clinical nutrition 2007;61(11):131822.

7. Davalos A, Ricart W, Gonzalez-Huix F, et al. Effect of malnutrition after acute stroke on clinical outcome. Stroke; a journal of cerebral circulation 1996;27(6):1028-32.

8. Gariballa SE, Parker SG, Taub N, Castleden CM. Influence of nutritional status on clinical outcome after acute stroke. Am J Clin Nutr 1998;68(2):275-81.

9. Behrouz R, Godoy DA, Topel CH, et al. Early Hypoalbuminemia is an Independent Predictor of Mortality in Aneurysmal Subarachnoid Hemorrhage. Neurocritical care 2016. 
10. Ibrahim GM, Macdonald RL. The network topology of aneurysmal subarachnoid haemorrhage. Journal of neurology, neurosurgery, and psychiatry 2015;86(8):895-901.

11. Limaye K, Yang JD, Hinduja A. Role of admission serum albumin levels in patients with intracerebral hemorrhage. Acta neurologica Belgica 2016;116(1):27-30.

12. Chen S, Yang Q, Chen G, Zhang JH. An update on inflammation in the acute phase of intracerebral hemorrhage. Translational stroke research 2015;6(1):4-8.

13. Boehme AK, Hays AN, Kicielinski KP, et al. Systemic Inflammatory Response Syndrome and Outcomes in Intracerebral Hemorrhage. Neurocritical care 2016.

14. Margarson MP, Soni N. Serum albumin: touchstone or totem? Anaesthesia 1998;53(8):789-803.

15. Behrouz R, Azarpazhooh MR, Godoy DA, et al. The Multi-National survey on Epidemiology, Morbidity, and Outcomes iN Intracerebral Haemorrhage (MNEMONICH). International journal of stroke : official journal of the International Stroke Society 2015;10(8):E86.

16. Di Napoli M, Zha AM, Godoy DA, et al. Prior Cannabis Use Is Associated with Outcome after Intracerebral Hemorrhage. Cerebrovascular diseases 2016;41(5-6):248-55.

17. Steiner T, Kaste M, Forsting M, et al. Recommendations for the management of intracranial haemorrhage - part I: spontaneous intracerebral haemorrhage. The European Stroke Initiative Writing Committee and the Writing Committee for the EUSI Executive Committee. Cerebrovascular diseases 2006;22(4):294-316.

18. Morgenstern LB, Hemphill JC, 3rd, Anderson C, et al. Guidelines for the management of spontaneous intracerebral hemorrhage: a guideline for healthcare professionals from the American Heart Association/American Stroke Association. Stroke 2010;41(9):2108-29.

19. Steiner T, Al-Shahi Salman R, Beer R, et al. European Stroke Organisation (ESO) guidelines for the management of spontaneous intracerebral hemorrhage. International journal of stroke : official journal of the International Stroke Society 2014;9(7):840-55. 
20. Kothari RU, Brott T, Broderick JP, et al. The ABCs of measuring intracerebral hemorrhage volumes. Stroke; a journal of cerebral circulation 1996;27(8):1304-5.

21. Brackeen GL, Dover JS, Long CL. Serum albumin. Differences in assay specificity. Nutr Clin Pract 1989;4(6):203-5.

22. Singer M, Deutschman CS, Seymour CW, et al. The Third International Consensus Definitions for Sepsis and Septic Shock (Sepsis-3). JAMA 2016;315(8):801-10.

23. van Swieten JC, Koudstaal PJ, Visser MC, Schouten HJ, van Gijn J. Interobserver agreement for the assessment of handicap in stroke patients. Stroke; a journal of cerebral circulation 1988;19(5):604-7.

24. Muller R, Buttner P. A critical discussion of intraclass correlation coefficients. Statistics in medicine 1994;13(23-24):2465-76.

25. Bath PM, Lees KR, Schellinger PD, et al. Statistical analysis of the primary outcome in acute stroke trials. Stroke; a journal of cerebral circulation 2012;43(4):1171-8.

26. Arroyo V, Garcia-Martinez R, Salvatella X. Human serum albumin, systemic inflammation, and cirrhosis. J Hepatol 2014;61(2):396-407.

27. Artigas A, Wernerman J, Arroyo V, Vincent JL, Levy M. Role of albumin in diseases associated with severe systemic inflammation: Pathophysiologic and clinical evidence in sepsis and in decompensated cirrhosis. J Crit Care 2015.

28. Carfray A, Patel K, Whitaker P, Garrick P, Griffiths GJ, Warwick GL. Albumin as an outcome measure in haemodialysis in patients: the effect of variation in assay method. Nephrol Dial Transplant 2000;15(11):1819-22.

29. McPherson RA, editor, Pincus MR, editor. Henry's clinical diagnosis and management by laboratory methods. 22 ed. Philadelphia, PA: Elsevier/Saunders, 2001. 
30. Hennig B, Honchel R, Goldblum SE, McClain CJ. Tumor necrosis factor-mediated hypoalbuminemia in rabbits. J Nutr 1988;118(12):1586-90.

31. McClain CJ, Hennig B, Ott LG, Goldblum S, Young AB. Mechanisms and implications of hypoalbuminemia in head-injured patients. J Neurosurg 1988;69(3):386-92.

32. Arima H, Wang JG, Huang Y, et al. Significance of perihematomal edema in acute intracerebral hemorrhage: the INTERACT trial. Neurology 2009;73(23):1963-8.

33. Di Napoli M, Godoy DA, Campi V, et al. C-reactive protein in intracerebral hemorrhage: time course, tissue localization, and prognosis. Neurology 2012;79(7):690-9.

34. Wang J, Dore S. Inflammation after intracerebral hemorrhage. J Cereb Blood Flow Metab 2007;27(5):894-908.

35. American College of Chest Physicians/Society of Critical Care Medicine Consensus Conference: definitions for sepsis and organ failure and guidelines for the use of innovative therapies in sepsis. Critical care medicine 1992;20(6):864-74.

36. Belayev L, Obenaus A, Zhao W, et al. Experimental intracerebral hematoma in the rat: characterization by sequential magnetic resonance imaging, behavior, and histopathology. Effect of albumin therapy. Brain Res 2007;1157:146-55.

37. Belayev L, Saul I, Busto R, et al. Albumin treatment reduces neurological deficit and protects blood-brain barrier integrity after acute intracortical hematoma in the rat. Stroke; a journal of cerebral circulation 2005;36(2):326-31.

38. Ginsberg MD, Palesch YY, Martin RH, et al. The albumin in acute stroke (ALIAS) multicenter clinical trial: safety analysis of part 1 and rationale and design of part 2. Stroke; a journal of cerebral circulation 2011;42(1):119-27.

39. Human albumin administration in critically ill patients: systematic review of randomised controlled trials. BMJ 1998;317(7153):235-40. 
40. Wilkes MM, Navickis RJ. Patient survival after human albumin administration. A meta-analysis of randomized, controlled trials. Ann Intern Med 2001;135(3):149-64. 


\section{FIGURE LEGENDS}

Figure 1 - Percentage distribution of patients with or without SIRS according to albumin quartile (a). Mean albumin values according to the presence of SIRS criteria (b). SIRS indicates systemic inflammatory response syndrome.

Figure 2 - Comparison of hospital discharge modified Rankin Scale scores between intracerebral hemorrhage patients with and without hypoalbuminemia. The distribution of scores on the modified Rankin scale is shown. Scores range from 0 to 6 , with 0 indicating no symptoms, 1 no clinically significant disability, 2 slight disability (patient is able to look after own affairs without assistance but is unable to carry out all previous activities), 3 moderate disability (patient requires some help but is able to walk unassisted), 4 moderately severe disability (patient is unable to attend to bodily needs without assistance and unable to walk unassisted), 5 severe disability (patient requires constant nursing care and attention), and 6 death. 
Table 1. Baseline characteristics of the cohort based on presence or absence of hypoalbuminemia (lower standardized albumin quartile).

\begin{tabular}{|c|c|c|c|c|c|}
\hline Characteristics & $\begin{array}{c}\text { No. of } \\
\text { observations }\end{array}$ & $\begin{array}{c}\text { Total cohort } \\
(\mathrm{n}=518)\end{array}$ & $\begin{array}{c}\text { Albumin } \\
<\text { lower quartile } \\
(\mathrm{n}=130)\end{array}$ & $\begin{array}{c}\text { Albumin } \\
\geq \text { lower quartile } \\
(\mathrm{n}=388)\end{array}$ & $P$ \\
\hline \multicolumn{6}{|l|}{ Demographic } \\
\hline Age, $\mathrm{y} \pm \mathrm{SD}$ & 518 & $68 \pm 13$ & $69 \pm 14$ & $69 \pm 12$ & 0.109 \\
\hline Female, n (\%) & 518 & $205(39.6)$ & $41(31.5)$ & $164(42.3)$ & 0.030 \\
\hline \multicolumn{6}{|l|}{ Clinical } \\
\hline Arterial hypertension, $\mathrm{n}(\%)$ & 484 & $391(80.8)$ & $100(82.6)$ & $29(80.2)$ & 0.549 \\
\hline Smoking (current or prior), $\mathrm{n}(\%)$ & 467 & $134(28.7)$ & $33(28.0)$ & $101(28.9)$ & 0.840 \\
\hline Alcohol regular use, n (\%) & 463 & $119(25.7)$ & $35(30.2)$ & $84(24.2)$ & 0.203 \\
\hline GCS (IQR) & 518 & $13(7-14)$ & $9(4-14)$ & $13(10-15)$ & $<0.0001$ \\
\hline ICH score (IQR) & 518 & $2(1-3)$ & $2(1-4)$ & $1(0-3)$ & $<0.0001$ \\
\hline SIRS at admission, n (\%) & 518 & $129(24.9)$ & $54(41.5)$ & $75(19.3)$ & $<0.0001$ \\
\hline Time-to-door (minutes) & 504 & $140(65-260)$ & $180(68-239)$ & $140(70-250)$ & 0.935 \\
\hline \multicolumn{6}{|l|}{ Imaging } \\
\hline Hematoma volume $\mathrm{mL}$ (IQR) & 517 & $18(9-37)$ & $30(12-66)$ & $15(8-33)$ & $<0.0001$ \\
\hline - $\quad<30 \mathrm{~mL}, \mathrm{n}(\%)$ & & $351(67.9)$ & $71(55.0)$ & $280(72.2)$ & $<0.0001$ \\
\hline - $\quad 30-60 \mathrm{~mL}, \mathrm{n}(\%)$ & & $84(16.2)$ & $26(20.2)$ & $58(14.9)$ & \\
\hline - $\quad>60 \mathrm{~mL}, \mathrm{n}(\%)$ & & $82(15.9)$ & $32(24.8)$ & $50(12.9)$ & \\
\hline IVH, n (\%) & 518 & $244(47.1)$ & $77(59.2)$ & $167(43.0)$ & $<0.0001$ \\
\hline Infratentorial, n (\%) & 518 & $69(13.3)$ & $7(5.4)$ & $62(16.0)$ & 0.0002 \\
\hline Location & 518 & & & & 0.049 \\
\hline Deep, n (\%) & & $304(58.7)$ & $85(65.4)$ & $219(56.4)$ & \\
\hline Lobar, n (\%) & & $143(27.6)$ & $37(28.5)$ & $106(27.3)$ & \\
\hline Midbrain, n (\%) & & $2(0.4)$ & - & $2(0.5)$ & \\
\hline Pons, n (\%) & & $24(4.6)$ & $1(0.8)$ & $23(5.9)$ & \\
\hline Cerebellum, n (\%) & & $43(8.3)$ & $6(4.6)$ & $37(9.5)$ & \\
\hline Primary IVH, n (\%) & & $2(0.4)$ & $1(0.8)$ & $1(0.3)$ & \\
\hline \multicolumn{6}{|l|}{ Laboratory } \\
\hline Standardized albumin,* (IQR) & 518 & $3.1(2.3-3.8)$ & $1.8(1.3-2.1)$ & $3.6(2.9-3.9)$ & $<0.0001$ \\
\hline Glucose, $\mathrm{mmol} / \mathrm{L}(\mathrm{IQR})$ & 515 & $7.8(6.2-10.8)$ & $8.1(6.4-11.5)$ & $7.4(6.2-10.4)$ & 0.164 \\
\hline C-reactive protein, $\mathrm{mg} / \mathrm{L}(\mathrm{IQR})$ & 501 & $8.7(4.0-19.0)$ & $10(5.0-21.0)$ & $8.4(6.0-17.0)$ & 0.070 \\
\hline WBC, $\times 10^{9} / \mathrm{L}(\mathrm{IQR})$ & 518 & $8.8(6.8-11.6)$ & $10.5(7.8-15.2)$ & $8.7(6.6-10.9)$ & $<0.0001$ \\
\hline Platelet x $10^{9} / \mathrm{L}(\mathrm{IQR})$ & 504 & $225(198-287)$ & $226(198-304)$ & $225(199-287)$ & 0.465 \\
\hline INR (IQR) & 510 & $1.16(1.10-1.25)$ & $1.21(1.12-1.35)$ & $1.16(1.10-1.23)$ & 0.408 \\
\hline \multicolumn{6}{|l|}{ Hemodynamics at admission } \\
\hline SBP, $\mathrm{mm} \mathrm{Hg}$ & 499 & $176 \pm 34$ & $180 \pm 35$ & $175 \pm 34$ & 0.878 \\
\hline $\mathrm{DBP}, \mathrm{mm} \mathrm{Hg}$ & 499 & $95 \pm 19$ & $95 \pm 20$ & $95 \pm 18$ & 0.229 \\
\hline $\mathrm{PP}, \mathrm{mmHg}$ & 499 & $81 \pm 22$ & $85 \pm 23$ & $80 \pm 22$ & 0.577 \\
\hline MAP, $\mathrm{mm} \mathrm{Hg}$ & 499 & $121 \pm 22$ & $123 \pm 23$ & $122 \pm 22$ & 0.910 \\
\hline \multicolumn{6}{|l|}{ Medications } \\
\hline Antiplatelet use, n (\%) & 518 & $183(35.3)$ & $50(38.5)$ & $133(34.3)$ & 0.388 \\
\hline Anticoagulant use, n (\%) & 518 & $44(8.5)$ & $17(13.1)$ & $27(7.0)$ & 0.030 \\
\hline
\end{tabular}

DBP diastolic blood pressure; GCS, Glasgow coma scale; ICH, intracerebral hemorrhage; INR, international normalized ratio; IQR, interquartile range; IVH, intraventricular hemorrhage; MAP, mean arterial pressure; 
PP, pulse pressure; SBP, systolic blood pressure, SD, standard deviation; SIRS, systemic inflammatory response syndrome; WBC, white blood cell count. *Standardized albumin values are obtained calculating the Z-score value for each independent cohort, by subtracting the mean and dividing by the standard deviation, and adding 3 units to each value in order to avoid negative results. 
Table 2. Multifactorial general linear regression analysis with continuous standardized albumin concentration as dependent variable. Odds ratio (OR) is calculated for one unit standardized albumin concentration decrease.

\begin{tabular}{|c|c|c|}
\hline Characteristics & OR & $(95 \% \mathrm{CI})$ \\
\hline Age (1 year increase) & 0.99 & $0.97-1.01$ \\
\hline Sex (female) & 1.20 & $1.02-1.42$ \\
\hline GCS & & $1.01-1.07$ \\
\hline Infratentorial & 0.66 & $0.52-0.85$ \\
\hline IVH & & $0.87-1.25$ \\
\hline SIRS & 1.40 & $1.11-1.76$ \\
\hline ICH Volume ${ }^{*}$ & 0.99 & $0.90-1.09$ \\
\hline INR at admission ${ }^{*}$ & 1.06 & $0.83-1.35$ \\
\hline Glucose at admission ${ }^{*}$ & 1.06 & $0.84-1.83$ \\
\hline
\end{tabular}

OR, Odds ratio; other abbreviations as per Table $1 .^{*}$ Log transformed 
Table 3. Association between all-cause mortality, hypoalbuminemia, and SIRS status.

\begin{tabular}{|c|c|c|c|c|}
\hline Clinical Outcome & $\begin{array}{c}\text { Albumin } \\
<\text { lower quartile }\end{array}$ & $\begin{array}{l}\text { Albumin } \\
\geq \text { lower quartile }\end{array}$ & SIRS - & SIRS + \\
\hline $\mathrm{n}$ & 130 & 388 & 389 & \\
\hline In-hospital mortality, n (\%) & $67(51.5)$ & $105(27.1)$ & $68(17.5)$ & $104(80.6)$ \\
\hline \multicolumn{5}{|l|}{ Univariate } \\
\hline OR $(95 \% \mathrm{CI})$ & $2.87(1.90-4.32)$ & 1.0 (reference) & 1.0 (reference) & $19.64(11.80-32.67)$ \\
\hline \multicolumn{5}{|l|}{ Multivariable } \\
\hline \multicolumn{5}{|l|}{ Model 1* } \\
\hline OR (95\% CI) & $2.00(1.21-3.33)$ & 1.0 (reference) & 1.0 (reference) & $19.18(11.33-32.46)$ \\
\hline \multicolumn{5}{|l|}{ Model $2 \dagger$} \\
\hline OR (95\% CI) & $1.30(0.50-3.36)$ & 1.0 (reference) & 1.0 (reference) & $5.52(2.29-13.32)$ \\
\hline
\end{tabular}

*Model 1 Age-and sex- adjusted

$\uparrow$ Model 2 adjusted for age, log-transformed ICH volume, INR, and GCS as continuous variables, and gender, infratentorial location, intraventricular extension, and SIRS at admission as binary variables and interaction between albumin and SIRS at admission.

OR, Odds ratio; other abbreviations as per Table 1. 

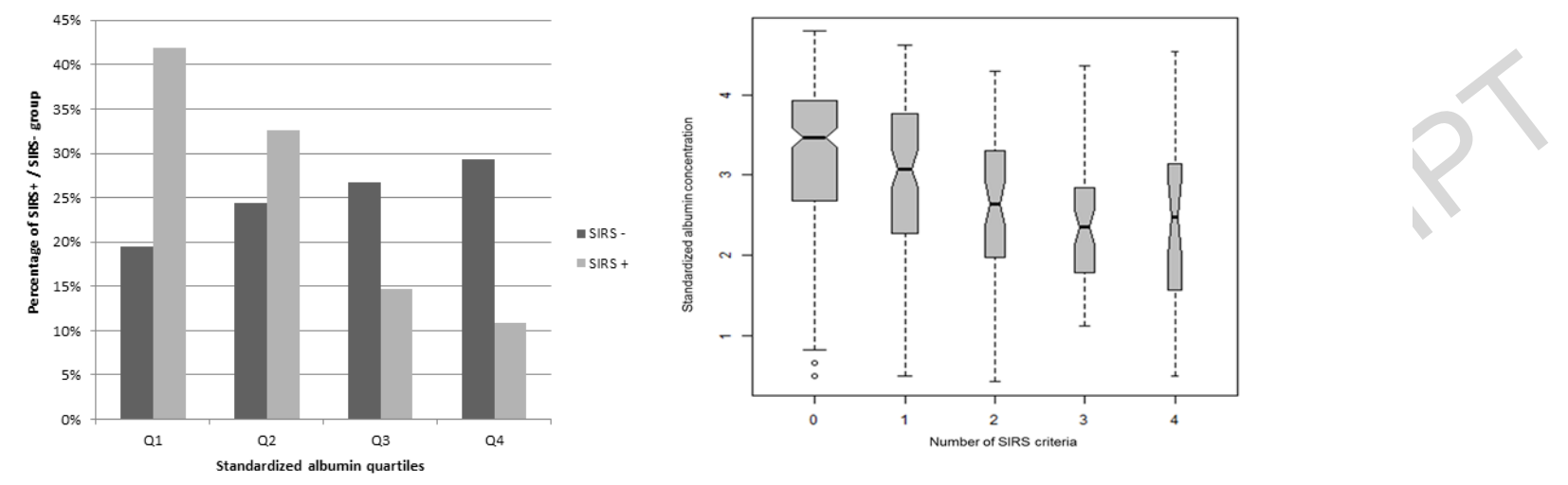

Figure 1

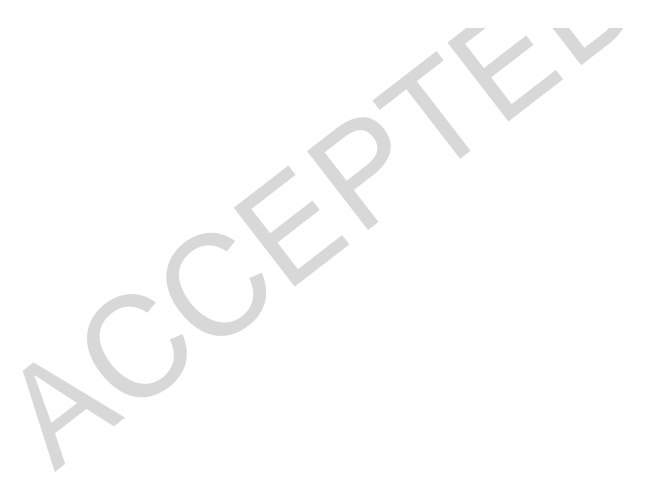




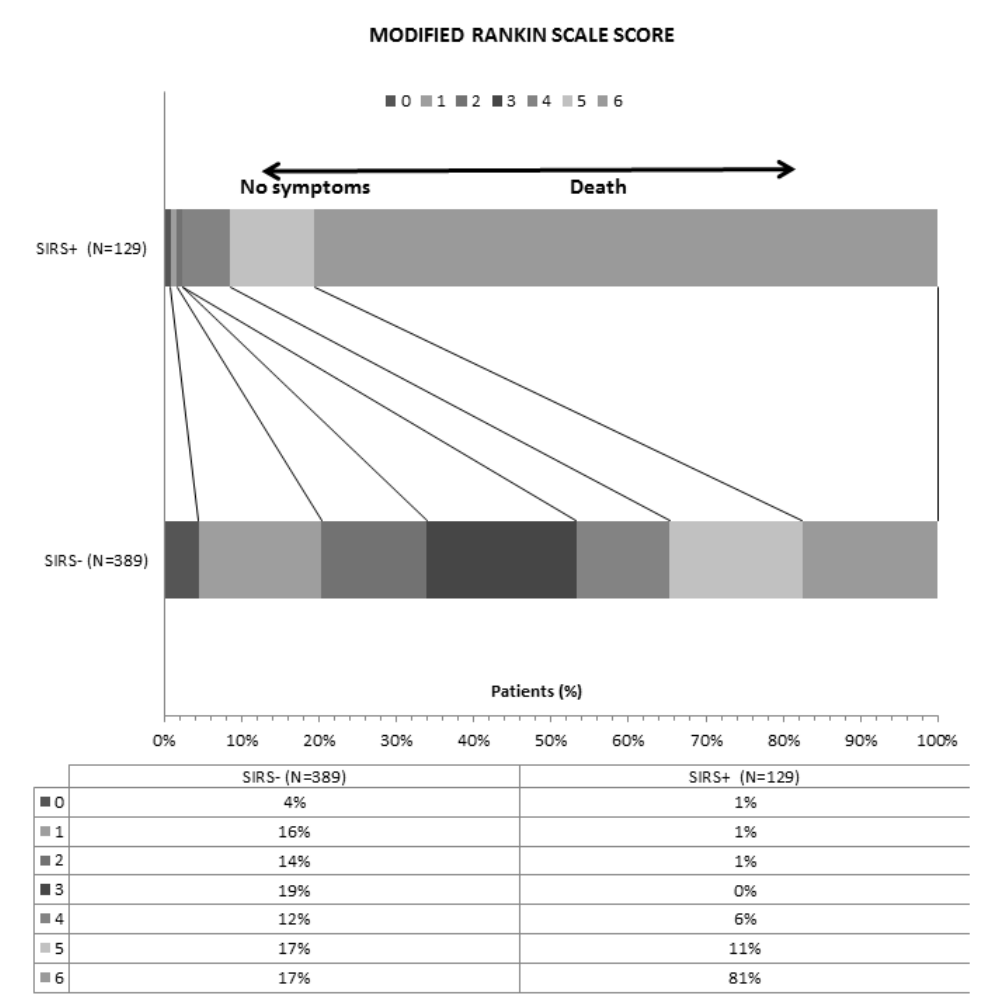

Figure 2

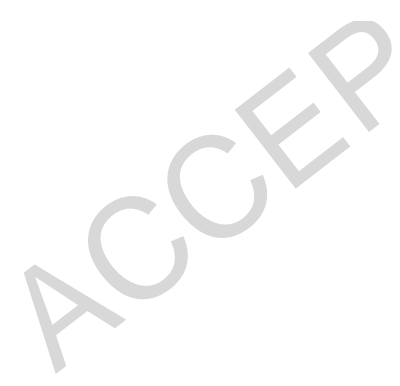

